\title{
A Note on Weak Solutions of Conservation Laws and Energy/Entropy Conservation
}

\author{
Piotr Gwiazda, Martin Michálek, \\ \& AgNiesZKa ŚwierCZEWSKA-GWIAZDA(D)
}

Communicated by P. Constantin

\begin{abstract}
A common feature of systems of conservation laws of continuum physics is that they are endowed with natural companion laws which are in such cases most often related to the second law of thermodynamics. This observation easily generalizes to any symmetrizable system of conservation laws; they are endowed with nontrivial companion conservation laws, which are immediately satisfied by classical solutions. Not surprisingly, weak solutions may fail to satisfy companion laws, which are then often relaxed from equality to inequality and overtake the role of physical admissibility conditions for weak solutions. We want to answer the question: what is a critical regularity of weak solutions to a general system of conservation laws to satisfy an associated companion law as an equality? An archetypal example of such a result was derived for the incompressible Euler system in the context of Onsager's conjecture in the early nineties. This general result can serve as a simple criterion to numerous systems of mathematical physics to prescribe the regularity of solutions needed for an appropriate companion law to be satisfied.
\end{abstract}

\section{Introduction}

The current decade has been preoccupied with solving the famous conjecture of Onsager, which says that solutions to the incompressible Euler system conserve total kinetic energy as long as they are Hölder continuous with a Hölder exponent $\alpha>1 / 3$, and that otherwise they may dissipate the energy.

The ideas used to prove the celebrated Nash-Kuiper theorem appeared to have wide applicability in the context of fluid mechanics, and the incompressible Euler system in particular. Interestingly, the construction of weak solutions via the appropriate refinement of the method of convex integration allowed one to generate solutions with a regularity exactly as prescribed by Onsager that do not conserve the energy. We shall summarize in a sequel the recent achievements in this direction, however our main interest in the current paper is aimed at an analogue and 
generalization of the first part of Onsager's statement. The positive direction of this claim was solved by EYINK in [17] under a *-Hölder condition, as the authors call it, which is stronger than a usual Hölder continuity. Later, a straightforward argument stated in Besov spaces (which contains Hölder spaces) was published by Constantin ET AL., cf. [8], see also [7,16] for later studies. A sufficient regularity for the energy to be conserved has been established for a variety of models, including the incompressible inhomogeneous Euler system and the compressible Euler in [18], the incompressible inhomogeneous Navier-Stokes system in [24], compressible Navier-Stokes in [27] and equations of magnetohydrodynamics in [6].

The above list gives a flavor of how broad is the class of systems for which one can specify the regularity of weak solutions which provides the energy to be conserved. This motivates us, instead of developing tools for another dozen of systems, to look at general systems of conservation laws. Apparently one can prescribe the condition for weak solutions providing that, in addition to a conservation law, they will satisfy a companion conservation law. To make the statement more precise, let us consider a conservation law, not necessarily hyperbolic, in a general form

$$
\operatorname{div}_{X}(G(U(X))=0 \quad \text { for } X \in \mathscr{X}
$$

for an unknown (vector) function $U=U(X): \mathscr{X} \rightarrow \mathscr{O}$ and a given matrix field $G: \mathscr{O} \rightarrow \mathbb{M}^{n \times(k+1)}$. Let us assume that $\mathscr{O}$ and $\mathscr{X}$ are open sets, $\mathscr{X} \subseteq \mathbb{R}^{k+1}$ or $\mathscr{X} \subseteq \mathbb{R} \times \mathbb{T}^{k}$ and $\mathscr{O} \subseteq \mathbb{R}^{n}$, where $\mathbb{T}^{k}$ denotes the flat torus of dimension $k$ (imposing the periodic boundary conditions). We denote $X=\left(x_{0}, x_{1}, \ldots, x_{k}\right)^{T}$ as the standard coordinates over $\mathbb{R}^{k+1}$ or $\mathbb{R} \times \mathbb{T}^{k}$ and we consider over $\mathscr{O}$ the coordinates $Y=\left(y_{1}, \ldots, y_{n}\right)^{T}$ with respect to the canonical basis. For a matrix field $M=\left(M_{i, j}\right)_{i=1, \ldots, n, j=0, \ldots, k}, M_{i, j}: \mathbb{R}^{n} \rightarrow \mathbb{R}$, we denote $M_{j}$ the $j$-th column vector. Moreover, we use the standard definition

$$
\operatorname{div}_{X} M(X)=\sum_{j=0}^{k} \partial_{x_{j}} M_{j}(X)
$$

We denote by $D_{X}$ (respectively $\left.D_{Y}, D_{U}\right)$ the differential $\left(D_{X}=\left(\partial_{x_{0}}, \ldots, \partial_{x_{k}}\right)\right)$ with respect to variables $X$ (respectively $Y, U$ ).

Following the notation in [9] we shall say that a smooth function $Q: \mathscr{O} \rightarrow \mathbb{R}^{k+1}$ is a companion of $G$ if there exists a smooth function $\mathcal{B}: \mathscr{O} \rightarrow \mathbb{R}^{n}$ such that

$$
D_{U} Q_{j}(U)=\mathcal{B}(U) D_{U} G_{j}(U) \text { for all } U \in \mathscr{O}, j \in\{0, \ldots, k\} .
$$

Observe that for any classical solution $U$ of (1), we obtain

$$
\operatorname{div}_{X}(Q(U(X)))=0 \text { for } X \in \mathscr{X},
$$

where by a classical solution we mean a Lipschitz continuous vector field $U$ satisfying (1) for almost all $X \in \mathscr{X}$. Identity (3) is called a companion law associated with $G$ (see e.g. [9]). In many applications, which we partially recall in Section 4, some relevant companion laws are conservation of energy or conservation of entropy. Before we discuss the relations between weak solutions and companion laws, 
let us remark that it was observed by GoDUNOv [19] that systems of conservation laws are symmetrizable if and only if they are endowed with nontrivial companion laws.

We consider the standard definition of weak solutions to a conservation law:

Definition. We call the function $U: \mathscr{X} \rightarrow \mathscr{O}$ a weak solution to (1) if $G(U)$ is locally integrable in $\mathscr{X}$ and the equality

$$
\int_{\mathscr{X}} G(U(X)): D_{X} \psi(X) \mathrm{d} X=0
$$

holds for all smooth test functions $\psi: \mathscr{X} \rightarrow \mathbb{R}^{n}$ with a compact support in $\mathscr{X}$.

Analogously, we can define weak solutions to (3), however weak solutions of (1) may not necessarily also be weak solutions to (3). The main question we deal with in this paper reads as follows: what are sufficient conditions for a weak solution of (1) to also satisfy (3)?

Let us comment in more detail on results related to the question of energy conservation for weak solutions of some conservation laws. Both parts of Onsager's conjecture for the incompressible inviscid Euler system have been resolved. Due to recent results of ISETT [20] and BUCKMASTER ET AL. [5], we know there exist for $\beta<1 / 3$ solutions of the incompressible Euler equations of class $C^{\beta}\left([0, T] \times \mathbb{T}^{3}\right)$ which do not satisfy the energy equality. These results were preceded with a series of papers showing firstly existence of bounded ([11]), later continuous ([12]) and Hölder continuous ([13]) solutions with $\alpha=1 / 10$. The further results aimed to increase the Hölder exponent; see [2-4,21].

In the context of our studies, the second part of Onsager's conjecture is more relevant. The first result was due to EYINK [17], however the author required stronger regularity. Concretely Eyink required that at all time the velocity field $u$ belongs to the space $C_{*}^{\alpha}\left(\mathbb{T}^{d}\right), \alpha>\frac{1}{3}$, of functions, whose Fourier coefficients satisfy the summability condition

$$
\sum_{k \in \mathbb{Z}^{d}}|k|^{\alpha}|\hat{u}(k)|<\infty .
$$

This condition can be easily seen to imply Hölder continuity of order $\alpha$, i.e. $C_{*}^{\alpha} \subset$ $C^{\alpha}$. However there is no equality; for example the function

$$
u(x)=\sum_{n=1}^{\infty} \frac{\cos \left(3^{n} x\right)}{3^{\alpha n}}
$$

is of class $C^{\alpha}\left(\mathbb{T}^{d}\right)$ for any $0<\alpha \leq 1$, but not $C_{*}^{\alpha}\left(\mathbb{T}^{d}\right)$.

CONSTANTIN ET AL. [8] showed the conservation of the global kinetic energy if the velocity field $u$ is of the class $L^{3}\left(0, T ; B_{3, \infty}^{\alpha}\left(\mathbb{T}^{3}\right)\right) \cap C\left([0, T] ; L^{2}\left(\mathbb{T}^{3}\right)\right)$ whenever $\alpha>\frac{1}{3}$, see also [17]. Here $B_{3, \infty}^{\alpha}$ stands for a Besov space (definition is recalled in Section 2). For the same system it was observed by CHESKIDOV ET AL. in [7] that it is sufficient for $u$ to belong to a larger space $L^{3}\left(0, T ; B_{3, q}^{1 / 3}\left(\mathbb{T}^{3}\right)\right)$ where $q \in(1, \infty)$. We refer the reader to $[25,26]$ for more refinements in the case of the incompressible 
Euler system. For the incompressible inviscid equations of magnetohydrodynamics, a result comparable to [8] was proved by CAFLISCH ET AL. [6], see also [22].

The standard technique developed in [8] is based on the convolution of the Euler system with a standard family of mollifiers. The crucial part of the proof is then to estimate an appropriate nonlinear commutator. Most of the mentioned results have been derived for systems with bilinear nonlinearity.

Recently, similar results for the compressible Euler system were presented by FEIREISL ET AL. in [18]. A sufficient condition for the energy conservation is that the solution belongs to $B_{3, \infty}^{\alpha}\left((0, T) \times \mathbb{T}^{3}\right)$ with $\alpha>1 / 3$. To the best of our knowledge, this was the first result treating nonlinearity which is not in a multilinear form. We extend this approach to a general class of conservation laws of the form (1). Let us mention that we are not aware of any reference where the problem would be treated in such generality. We believe that this general scenario might be of interest. Moreover, at least the application on the equations of polyconvex elastodynamics (Subsection 4.3) is an original contribution of this paper.

Let us present the main results of the paper. For the notation, we refer the reader to Section 2.

Theorem 1.1. Let $U \in B_{3, \infty}^{\alpha}(\mathscr{X} ; \mathscr{O})$ be a weak solution of (1) with $\alpha>\frac{1}{3}$. Assume that $G \in C^{2}\left(\mathscr{O} ; \mathbb{M}^{n \times(k+1)}\right)$ is endowed with a companion law with flux $Q \in C\left(\mathscr{O} ; \mathbb{R}^{k+1}\right)$ for which there exists $\mathcal{B} \in C^{1}\left(\mathscr{O} ; \mathbb{R}^{n}\right)$ related through identity (2) and all the following conditions hold:

$$
\left.\begin{array}{r}
\mathscr{O} \text { is convex, } \\
\mathcal{B} \in W^{1, \infty}\left(\mathscr{O} ; \mathbb{R}^{n}\right), \\
\sup _{i, j \in 1, \ldots, n}\left\|\partial_{U_{i}} \partial_{U_{j}} G(U)\right\|_{C\left(\mathscr{O} ; \mathbb{M}^{n \times(k+1)}\right)}<+\infty .
\end{array}\right\}
$$

Then $U$ is a weak solution of the companion law (3) with the flux $Q$.

Remark. - We can in the same manner treat companion laws which are not a scalar equation (i.e., $Q: \mathscr{O} \rightarrow \mathbb{M}^{s \times(k+1)}, \mathcal{B}: \mathscr{O} \rightarrow \mathbb{M}^{s \times n}$ and $s>1$ ). In such case we apply Theorem 1.1 to each row of (3).

- The growth condition of $Q$ can be relaxed whenever $B_{3, \infty}^{\alpha}$ is embedded to an appropriate Lebesgue space.

- Under suitable assumptions, one can extend the theory on non-homogeneous fluxes $G=G(X, U)$ and Equation (1) with non-zero right-hand side $h=$ $h(X, U)$.

- Due to the definition of weak solutions, it is enough to consider the integrability and regularity of $U$ only locally in $\mathscr{X}$.

Due to the assumption on the convexity of $\mathscr{O}$, Theorem 1.1 could be straightforwardly deduced from [18], however, for the reader's convenience, we present the proof in Section 3. It is worth noting that the convexity of $\mathscr{O}$ might not be natural for all applications (this is e.g. the case of the polyconvex elasticity, see Section 4.) To this end, we present a theorem dealing with the case of non-convex $\mathscr{O}$. 
Theorem 1.2. Let the assumptions of Theorem 1.1 be satisfied, but instead of (6) we assume that

the essential range of $U$ is compact in $\mathscr{O}$.

Then $U$ is a weak solution of the companion law (3) with the flux $Q$.

Apparently, the conclusions of the previous theorems are reasonably weaker in comparison with some known results for particular conservation laws. As an example, the result of Constantin ET AL. in [8] does not need the Besov-type regularity with respect to time. Having more knowledge about the nonlinear part of $G$, we may be able to relax the class of solutions in Theorem 1.1, what is discussed in Section 4.

The main result, following the methods of [7], could be formulated in a sharper version, where we would reach the exponent $\alpha=1 / 3$. We decided to omit this formulation, for simplicity of presentation. In fact the cost to pay for the exact exponent $1 / 3$ needs delicately better summability, as the assumed Besov regularity would not be $B_{3, \infty}^{1 / 3}$, but $B_{3, c_{0}}^{1 / 3}$ (which is a larger space). We omit here the definition, and refer the interested reader to [7] for details and just mention that an appropriate test function needs to be chosen in terms of a Littlewood-Paley decomposition. Even more interestingly, as was mentioned in Remark 3.3. in [24], the condition $u \in L^{3}\left(0, T ; B_{3, c_{0}}^{1 / 3}\right)$ could be replaced by a weaker condition

$$
\lim _{y \rightarrow 0} \frac{1}{|y|} \int_{\mathbb{T}^{d} \times[0, T]}|u(t, x+y)-u(t, x)|^{3} \mathrm{~d} x \mathrm{~d} t=0 .
$$

For the case of a general conservation law the sufficient condition should concern a shift in all the variables unless some additional structure of linearity is known.

Finally, we observe that in case we consider hyperbolic systems, the opposite direction of the Onsager's hypothesis is almost trivial. This is of course a completely different situation to the case of incompressible Euler system, which is not a hyperbolic conservation law and where the construction of solutions dissipating the energy was a challenge. It is well known, cf. [9, Chapter 1] among others, that shock solutions dissipate energy. Following Dafermos again, we note that crucial properties of local behavior of shocks may be investigated, without loss of generality, within the framework of systems in one-space dimension. Thus the essence can be already seen even on a simple example of Burger's equation $u_{t}+\left(u^{2} / 2\right)_{x}=0$. Classical solutions also satisfy $\left(u^{2} / 2\right)_{t}+\left(u^{3} / 3\right)_{x}=0$, which can be considered as a companion law. The shock solutions to the equation in the first form satisfy Rankine-Hugoniot condition $s\left(u_{l}-u r\right)=\left(u_{l}^{2}-u_{r}^{2}\right) / 2$, thus the speed of the shock is $s=\left(u_{l}+u_{r}\right) / 2$, where $u_{l}=\lim _{y \rightarrow x(t)^{-}} u(y, t)$ and $u_{r}$ is defined correspondingly. Considering the second equation one gets $s=2\left(u_{l}^{2}+u_{l} u_{r}+u_{r}^{2}\right) / 3\left(u_{l}+u_{r}\right)$, which in an obvious way is different. More generally, if we multiply (1) with the function $\mathcal{B}$ one easily concludes that to provide Rankine-Hugoniot conditions to be satisfied for the companion law, we end up with a trivial companion law, namely $\mathcal{B} \equiv$ const . 
Thus, knowing the regularity of shock solutions, as was shown in [18, Proposition 2.1], we have

$$
\left(B V \cap L^{\infty}\right)(\Omega) \subset B_{\infty}^{\frac{1}{q}}(\Omega)
$$

for every $q \in[1,+\infty]$; we observe that our assumptions are sharp.

Let us briefly mention the outline of the rest of the paper. In Section 2, we introduce the notation. Section 3 contains proofs of the main propositions. Section 4 is devoted to some relaxation of the conditions in Theorem 1.1 and applications of the main theorems are also presented.

\section{Notation and Auxiliary Estimates}

We will briefly present some properties of the Besov spaces $B_{q, \infty}^{\alpha}$. Let $\mathscr{X}$ be as above, $\alpha \in(0,1)$ and $q \in[1, \infty)$. We denote by $B_{q, \infty}^{\alpha}(\mathscr{X})$ the Besov space which is defined as follows:

$$
B_{q, \infty}^{\alpha}(\mathscr{X})=\left\{U \in L^{q}(\mathscr{X}): \quad|U|_{B_{q, \infty}^{\alpha}(\mathscr{X})}<\infty\right\},
$$

with

$$
|U|_{B_{q, \infty}^{\alpha}(\mathscr{X})}=\sup _{\xi \in \mathbb{R}^{k}} \frac{\|U(\cdot)-U(\cdot-\xi)\|_{L^{q}(\mathscr{X} \cap(\mathscr{X}+\xi))}}{|\xi|^{\alpha}} .
$$

On $B_{q, \infty}^{\alpha}(\mathscr{X})$ we consider the standard norm

$$
\|U\|_{B_{q, \infty}^{\alpha}(\mathscr{X})}^{q}=\|U\|_{L^{q}(\mathscr{X})}^{q}+|U|_{B_{q, \infty}^{\alpha}(\mathscr{X})}^{q} .
$$

Assume that a non-negative function $\eta_{1} \in C^{\infty}\left(\mathbb{R}^{k}\right)$ has a compact support in $B(0,1)$ and $\int_{\mathbb{R}^{k}} \eta_{1}(X) \mathrm{d} X=1$. For $\varepsilon>0$ we denote $\eta_{\varepsilon}(X)=\frac{1}{\varepsilon^{k}} \eta_{1}\left(\frac{X}{\varepsilon}\right)$ and

$$
[f]_{\varepsilon}(X)=f * \eta_{\varepsilon}(X),
$$

which is defined at least in $\mathscr{X}_{\varepsilon}=\{X \in \mathscr{X}: \operatorname{dist}(X, \partial \mathscr{X})>\varepsilon\}$. For vector or matrix-valued functions the convolution is defined component-wise. For $\mathscr{K} \subseteq \mathbb{R}^{k}$ and $\delta>0$ we also use the notation

$$
\mathscr{K}^{\delta}=\left\{X \in \mathbb{R}^{k}: \operatorname{dist}(X, \mathscr{K})<\delta\right\}=\cup_{X \in \mathscr{K}} B(X, \delta) .
$$

One easily shows that for $f \in B_{q, \infty}^{\alpha}(\mathscr{X})$ the following estimates hold:

$$
\begin{aligned}
\left\|D_{X}[f]_{\varepsilon}\right\|_{L^{q}\left(\mathscr{X}_{\varepsilon}\right)} & \leq C\|f\|_{B_{q, \infty}^{\alpha}(\mathscr{X})} \varepsilon^{\alpha-1}, \\
\left\|[f]_{\varepsilon}-f\right\|_{L^{q}\left(\mathscr{X}_{\varepsilon}\right)} & \leq C\|f\|_{B_{q, \infty}^{\alpha}(\mathscr{X})} \varepsilon^{\alpha}, \\
\|f(\cdot-y)-f(\cdot)\|_{L^{q}(\mathscr{X} \cap(\mathscr{X}+y))} & \leq C\|f\|_{B_{q, \infty}^{\alpha}(\mathscr{X})|y|^{\alpha},}
\end{aligned}
$$

where $C$ depends only on $\mathscr{X}$. 


\section{The Proof of the Main Results}

In what follows, we will denote by $C$ a constant independent of $\varepsilon$.

\subsection{Commutator Estimates}

The essential part of the proof of Theorem 1.1 pertains the estimation of the nonlinear commutator

$$
[G(U)]_{\varepsilon}-G\left([U]_{\varepsilon}\right) .
$$

This is based on the following observation, which appears in a special form in [18] (the rest of the proof of Theorem 1.1 is a reminiscence of the paper [8]):

Lemma 3.1. Let $\mathscr{O}$ be a convex set, $U \in L_{\text {loc }}^{2}(\mathscr{X} ; \mathscr{O}), G \in C^{2}\left(\mathscr{O} ; \mathbb{R}^{n}\right)$ and let

$$
\sup _{i, j \in 1, \ldots, n}\left\|\partial_{U_{i}} \partial_{U_{j}} G(U)\right\|_{L^{\infty}(\mathscr{O})}<+\infty .
$$

Then there exists $C>0$ depending only on $\eta_{1}$, second derivatives of $G$ and $k$ (dimension of $\mathscr{O}$ ) such that

$$
\begin{aligned}
& \left\|[G(U)]_{\varepsilon}-G\left([U]_{\varepsilon}\right)\right\|_{L^{q}(K)} \\
& \leq C\left(\left\|[U]_{\varepsilon}-U\right\|_{L^{2 q}(K)}^{2}+\sup _{Y \in \operatorname{supp} \eta_{\varepsilon}}\|U(\cdot)-U(\cdot-Y)\|_{L^{2 q}(K)}^{2}\right)
\end{aligned}
$$

for $q \in[1, \infty)$, where $K \subseteq \mathscr{X}$ satisfies $K^{\varepsilon} \subseteq \mathscr{X}$.

Proof. Without loss of generality, we assume that $G$ is a scalar function and $U$ is finite everywhere in $\mathscr{X}$. Then, because of (12) we get, for $X, Y \in K$,

$$
\begin{aligned}
& \left|G(U(X))-G\left([U]_{\varepsilon}(X)\right)-D_{U} G \circ U(X)\left(U(X)-[U]_{\varepsilon}(X)\right)\right| \\
& \quad \leq C\left|U(X)-[U]_{\varepsilon}(X)\right|^{2}, \\
& \left|G(U(X))-G(U(Y))-D_{U} G \circ U(X)(U(X)-U(Y))\right| \\
& \quad \leq C|U(X)-U(Y)|^{2} .
\end{aligned}
$$

We convolve (14) with $\eta_{\varepsilon}$ in variable $Y$ and apply Jensen's inequality on the lefthand side to get

$$
\begin{aligned}
& \left|G(U(X))-[G(U)]_{\varepsilon}(X)-D_{U} G \circ U(X)\left(U(X)-[U]_{\varepsilon}(X)\right)\right| \\
& \quad \leq C|U(X)-U(\cdot)|^{2} *_{Y} \eta_{\varepsilon} .
\end{aligned}
$$

Finally, coupling (13) and (15) implies

$$
\begin{aligned}
& \left|G\left([U]_{\varepsilon}(X)\right)-[G(U)]_{\varepsilon}(X)\right| \\
& \quad \leq C\left(\left|U(X)-[U]_{\varepsilon}(X)\right|^{2}+|U(X)-U(\cdot)|^{2} *_{Y} \eta_{\varepsilon}(X)\right) .
\end{aligned}
$$


In order to complete the proof, we use Jensen's inequality to estimate the $L^{q}$ norm of the second term on the right-hand side of (16) to get

$$
\begin{aligned}
& \int_{K}\left|\int_{\operatorname{supp} \eta_{\varepsilon}}\right| U(X)-\left.\left.U(X-Y)\right|^{2} \eta_{\varepsilon}(Y) \mathrm{d} Y\right|^{q} \mathrm{~d} X \\
& \quad \leq \int_{\operatorname{supp} \eta_{\varepsilon}} \int_{K}|U(X)-U(X-Y)|^{2 q} \eta_{\varepsilon}(Y) \mathrm{d} X \mathrm{~d} Y \\
& \quad \leq \sup _{Y \in \operatorname{supp} \eta_{\varepsilon}}\|U(\cdot)-U(\cdot-Y)\|_{L^{2 q}(K)}^{2 q} .
\end{aligned}
$$

\subsection{Proof of Theorem 1.1}

Let $\varepsilon_{0}>0$ and consider a test function $\psi \in C^{\infty}(\mathscr{X})$ such that supp $\psi \subseteq \mathscr{X}_{\varepsilon_{0}}$. Mollifying (1) by $\eta_{\varepsilon}$, we obtain

$$
\operatorname{div}_{X}[G(U)]_{\varepsilon}=0 \text { in } \mathscr{X}_{\varepsilon_{0}}
$$

whenever $\varepsilon<\varepsilon_{0}$. We multiply both sides of (17) by $\psi \mathcal{B}\left([U]_{\varepsilon}\right)$ [where $\mathcal{B}$ comes from (2)] from the left and get

$$
\int_{\mathscr{X}} \psi(X) \mathcal{B}\left([U]_{\varepsilon}(X)\right) \operatorname{div}_{X}\left([G(U)]_{\varepsilon}(X)\right) \mathrm{d} X=0
$$

We can recast the previous equality as follows:

$$
\int_{\mathscr{X}} \psi(X) \mathcal{B}\left([U]_{\varepsilon}(X)\right) \operatorname{div}_{X} G\left([U]_{\varepsilon}(X)\right) \mathrm{d} X=\int_{\mathscr{X}} R_{\varepsilon} \mathrm{d} X
$$

with the commutator

$$
R_{\varepsilon}=\psi(X) \mathcal{B}\left([U]_{\varepsilon}(X)\right) \operatorname{div}_{X}\left(G\left([U]_{\varepsilon}(X)\right)-[G(U)]_{\varepsilon}(X)\right) .
$$

Due to (2), equality (18) might be adjusted to the form

$$
-\int_{\mathscr{X}} Q\left([U]_{\varepsilon}(X)\right)\left(D_{X} \psi(X)\right)^{T} \mathrm{~d} X=\int_{\mathscr{X}} R_{\varepsilon} \mathrm{d} X .
$$

In order to show that the right-hand side of (20) converges to zero as $\varepsilon \rightarrow 0$, we write

$$
\begin{aligned}
\int_{\mathscr{X}} R_{\varepsilon}(X) \mathrm{d} X & =\int_{\mathscr{X}}\left(G\left([U]_{\varepsilon}\right)-[G(U)]_{\varepsilon}\right):\left(\left(D_{U} \mathcal{B}^{T}\right)\left([U]_{\varepsilon}\right) D_{X}[U]_{\varepsilon} \psi\right) \mathrm{d} X \\
& +\int_{\mathscr{X}}\left(G\left([U]_{\varepsilon}\right)-[G(U)]_{\varepsilon}\right):\left(\mathcal{B}^{T}\left([U]_{\varepsilon}\right) D_{X} \psi\right) \mathrm{d} X \\
& =I_{\varepsilon}^{1}+I_{\varepsilon}^{2} .
\end{aligned}
$$


The first integral is estimated using Lemma 3.1 and (9) as follows:

$$
\begin{aligned}
\left|I_{\varepsilon}^{2}\right| & \leq C\|\mathcal{B}\|_{W^{1, \infty}(\mathscr{O})}\left\|D_{X}[U]_{\varepsilon}\right\|_{L^{3}\left(\mathscr{X}_{\varepsilon_{0}}\right)}\left\|[U]_{\varepsilon}-U\right\|_{L^{3}\left(\mathscr{X}_{\varepsilon_{0}}\right)}^{2}\|\psi\|_{W^{1, \infty}\left(\mathscr{X}_{\varepsilon_{0}}\right)} \\
& \leq C \varepsilon^{\alpha-1} \varepsilon^{2 \alpha} .
\end{aligned}
$$

Similarly, we have

$$
\left|I_{\varepsilon}^{1}\right| \leq C \varepsilon^{\alpha}
$$

hence,

$$
\int_{\mathscr{X}} R_{\varepsilon} \mathrm{d} X \rightarrow 0 \text { as } \varepsilon \rightarrow 0
$$

as long as $\alpha>\frac{1}{3}$.

The convergence of the left-hand side of (20) follows from the Vitali theorem. Indeed, the equi-integrability of $Q\left([U]_{\varepsilon}\right)$ in $\mathscr{X}_{\varepsilon_{0}}$ is a consequence of that of $\left|[U]_{\varepsilon}\right|^{3}$ and the growth conditions on $Q$.

Remark. Having $\mathscr{O}$ non-convex, we face the problem that $[U]_{\varepsilon}$ does not have to belong to $\mathscr{O}$. The convexity was crucial to conduct the Taylor expansion argument in Lemma 3.1. However, we will see that a suitable extension of functions $G, \mathcal{B}$ and $Q$ does not alter the previous proof significantly.

\subsection{Proof of Theorem 1.2}

There exists $\delta>0$ depending only on $\mathscr{K}$ and $\mathscr{O}$ such that $\mathscr{K}^{2 \delta} \subseteq \mathscr{O}$. Let $\tilde{G} \in C^{2}\left(\mathbb{R}^{n} ; \mathbb{M}^{n \times(k+1)}\right), \tilde{\mathcal{B}} \in C^{1}\left(\mathbb{R}^{n} ; \mathbb{R}^{n}\right)$ and $\tilde{Q} \in C\left(\mathbb{R}^{n} ; \mathbb{R}^{k+1}\right)$ be compactly supported functions satisfying $\tilde{G}=G, \tilde{\mathcal{B}}=\mathcal{B}$ and $\tilde{Q}=Q$ in $\mathscr{K}^{\delta}$. Such functions exist as there is a set $\mathscr{R}$ with a smooth boundary satisfying $\mathscr{K}^{\delta} \subseteq \mathscr{R} \subseteq \mathscr{O}$. Thus, relation (2) holds also for $G, \mathcal{B}$ and $Q$ in $\mathscr{K}^{\delta}$.

In a fashion similar to the proof of Theorem 1.1 , for a function $\psi \in C^{\infty}(\mathscr{X})$ compactly supported in $\mathscr{X}_{\varepsilon_{0}}$, we obtain, for $\varepsilon<\varepsilon_{0}$,

$$
\int_{\mathscr{X}} \psi \tilde{\mathcal{B}}\left([U]_{\varepsilon}\right) \operatorname{div}_{X}[\tilde{G}(U)]_{\varepsilon} \mathrm{d} X=0 .
$$

We can write the previous equality as follows:

$$
\int_{\mathscr{X}} \psi \tilde{\mathcal{B}}\left([U]_{\varepsilon}\right) \operatorname{div}_{X} \tilde{G}\left([U]_{\varepsilon}\right) \mathrm{d} X=\int_{\mathscr{X}} \tilde{R}_{\varepsilon} \mathrm{d} X,
$$

with the commutator

$$
\tilde{R}_{\varepsilon}=\psi \tilde{\mathcal{B}}\left([U]_{\varepsilon}\right) \operatorname{div}_{X}\left(\tilde{G}\left([U]_{\varepsilon}\right)-[\tilde{G}(U)]_{\varepsilon}\right) .
$$

Analogously to Subsection 3.2, $\int_{\mathscr{X}} \tilde{R}_{\varepsilon} \mathrm{d} X$ vanishes as $\varepsilon \rightarrow 0$ due to Lemma 3.1, hence we may turn our attention to the left-hand side of (22); we show that it converges to

$$
-\int_{\mathscr{X}} Q(U)\left(D_{X} \psi\right)^{T} \mathrm{~d} X
$$


To this end, we put

$$
\mathscr{G}_{\varepsilon}^{\delta}=\left\{X \in \mathscr{X}:\left|U(X)-[U]_{\varepsilon}(X)\right|<\delta\right\},
$$

and since $D_{U} \tilde{Q}_{j}\left([U]_{\varepsilon}\right)=\tilde{\mathcal{B}}\left([U]_{\varepsilon}\right) D_{U} \tilde{G}_{j}\left([U]_{\varepsilon}\right)$ on $\mathscr{G}_{\varepsilon}^{\delta}$, we obtain

$$
\begin{aligned}
& \left|\int_{\mathscr{X}} \psi \tilde{\mathcal{B}}\left([U]_{\varepsilon}\right) \operatorname{div}_{X} \tilde{G}\left([U]_{\varepsilon}\right) \mathrm{d} X+\int_{\mathscr{X}} Q(U)\left(D_{X} \psi\right)^{T} \mathrm{~d} X\right| \\
& \quad \leq\left|\int_{\mathscr{X} \backslash \mathscr{G}_{\varepsilon} \delta} \psi \tilde{\mathcal{B}}\left([U]_{\varepsilon}\right) \operatorname{div}_{X} \tilde{G}\left([U]_{\varepsilon}\right) \mathrm{d} X\right|+\left|\int_{\mathscr{X} \backslash \mathscr{G}_{\varepsilon}^{\delta}} Q(U)\left(D_{X} \psi\right)^{T} \mathrm{~d} X\right| \\
& \quad+\left|\int_{\mathscr{G}_{\varepsilon}}\left(\tilde{Q}(U)-\tilde{Q}\left([U]_{\varepsilon}\right)\right)\left(D_{X} \psi\right)^{T} \mathrm{~d} X\right|=I_{\varepsilon}^{1}+I_{\varepsilon}^{2}+I_{\varepsilon}^{3} .
\end{aligned}
$$

To estimate $I_{\varepsilon}^{1}$, recall that $\tilde{G}$ and $\tilde{\mathcal{B}}$ are compactly supported, therefore

$$
\begin{aligned}
I_{\varepsilon}^{1} & \leq \int_{\mathscr{X} \backslash \mathscr{G}_{\varepsilon}^{\delta}}\left|\psi \tilde{\mathcal{B}}\left([U]_{\varepsilon}\right) D_{U} \tilde{G}\left([U]_{\varepsilon}\right) D_{X}[U]_{\varepsilon}\right| \mathrm{d} X \\
& \leq C\|\psi\|_{C^{1}} \int_{\mathscr{X} \backslash \mathscr{G}_{\varepsilon}^{\delta}}\left|D_{X}[U]_{\varepsilon}\right| \mathrm{d} X .
\end{aligned}
$$

By means of Hölder's and Chebyshev's inequalities, as well as (9) and (10), we observe that

$$
\begin{aligned}
I_{\varepsilon}^{1} & \leq C\|\psi\|_{C^{1}}\left\|D_{X}[U]_{\varepsilon}\right\|_{L^{3}\left(\mathscr{X}_{\varepsilon_{0}}\right)}\left|\mathscr{X} \backslash \mathscr{G}_{\varepsilon}^{\delta}\right|^{\frac{2}{3}} \\
& =C\|\psi\|_{C^{1}}\left\|D_{X}[U]_{\varepsilon}\right\|_{L^{3}\left(\mathscr{X}_{\varepsilon_{0}}\right)}\left|\left\{\left|U-[U]_{\varepsilon}\right|>\delta\right\}\right|^{\frac{2}{3}} \\
& \leq \frac{C\|\psi\|_{C^{1}}}{\delta^{2}}\left\|D_{X}[U]_{\varepsilon}\right\|_{L^{3}\left(\mathscr{X}_{\varepsilon_{0}}\right)}\left\|U-[U]_{\varepsilon}\right\|_{L^{3}\left(\mathscr{X}_{\varepsilon_{0}}\right)}^{2} \\
& \leq \frac{C\|\psi\|_{C^{1}}}{\delta^{2}} \varepsilon^{3 \alpha-1} .
\end{aligned}
$$

The integral $I_{\varepsilon}^{2}$ vanishes, as $\|Q(U)\|_{L^{\infty}(\mathscr{X})}<\infty$. Finally, we observe that

$$
I_{\varepsilon}^{3} \leq\|\psi\|_{C^{1}} \int_{\mathscr{X}_{\varepsilon_{0}}}\left|\tilde{Q}(U)-\tilde{Q}\left([U]_{\varepsilon}\right)\right| \mathrm{d} X .
$$

Therefore, $I_{\varepsilon}^{3} \rightarrow 0$ due to the almost everywhere convergence of $\tilde{Q}(U)-\tilde{Q}\left([U]_{\varepsilon}\right)$ to zero and boundedness of $\tilde{Q}$.

\section{Applications}

Observe that we have considered so far genuinely nonlinear fluxes $G$. The key part of the proof was to estimate

$$
\int_{\mathscr{X}}\left(G\left([U]_{\varepsilon}\right)-[G(U)]_{\varepsilon}\right):\left(\left(D_{U} \mathcal{B}^{T}\right)\left([U]_{\varepsilon}\right) D_{X}[U]_{\varepsilon} \psi\right) \mathrm{d} X,
$$


where the integral vanishes whenever $G$ is affine. Using this observation we might expect to drop some conditions on $U$ in the main theorems if some components of $G$ are affine functions.

We present three extensions of Theorem 1.1, which follow directly from the previous observation. The first gives a sufficient condition to drop the Besov regularity with respect to some variables. It is connected with the columns of $G$.

Corollary 4.1. Let $G=\left(G_{1}, \ldots, G_{s}, G_{s+1}, \ldots G_{k}\right)$ where $G_{1}, \ldots, G_{s}$ are affine vector-valued functions and $\mathscr{X}=\mathscr{Y} \times \mathscr{Z}$ where $\mathscr{Y} \subseteq \mathbb{R}^{s}$ and $\mathscr{Z} \subseteq \mathbb{R}^{k+1-s}$. Then it is enough to assume that $U \in L^{3}\left(\mathscr{Y} ; B_{3, \infty}^{\alpha}(\mathscr{Z})\right)$ in Theorem 1.1.

Next, we specify when we can omit the Besov regularity with respect to some components of $U$.

Corollary 4.2. Assume that $U=\left(V_{1}, V_{2}\right)$ where $V_{1}=\left(U_{1}, \ldots, U_{s}\right)$ and $V_{2}=$ $\left(U_{s+1}, \ldots, U_{n}\right)$. If $\mathcal{B}$ does not depend on $V_{1}$ and $G=G\left(V_{1}, V_{2}\right)=G_{1}\left(V_{1}\right)+$ $G_{2}\left(V_{2}\right)$ and $G_{1}$ is linear then it is enough to assume $U_{1}, \ldots, U_{s} \in L^{3}(\mathscr{X})$ in Theorem 1.1.

Finally, we deal with the case when some components of $\mathcal{B}$ are not Lipschitz in $\mathscr{O}$, but appropriate rows of $G$ are affine functions.

Corollary 4.3. Assume that a $j$-th row of $G$ is an affine function. Then the statement of Theorem 1.1 holds even if we assume that $\mathcal{B}_{j}$ is only locally Lipschitz in $\mathscr{O}$.

In the rest of this paper, we present a few examples on which the general theory applies. Some of them show how the general framework allows for the recovery of some known results. In what follows, we consider $\mathscr{X}=(0, T) \times \mathbb{T}^{3}, X=(t, x)$ and $\alpha>\frac{1}{3}$. We also present the systems in their standard form denoting $\nabla_{x}$ and $\operatorname{div}_{x}$ the correspondent operators with respect to the spatial coordinate $x$.

\subsection{Incompressible Euler System}

Let us consider the system of equations

$$
\left.\begin{array}{r}
\operatorname{div}_{x} \mathbf{u}^{T}=0 \\
\partial_{t} \mathbf{u}+\left(\mathbf{u} \cdot \nabla_{x}\right) \mathbf{u}+\nabla_{x} p=0
\end{array}\right\} \quad \text { in } \mathscr{X}
$$

for an unknown vector field $\mathbf{u}:(0, T) \times \mathbb{T}^{3} \rightarrow \mathbb{R}^{3}$ and scalar $p:(0, T) \times \mathbb{T}^{3} \rightarrow \mathbb{R}$. The system can be rewritten into the divergence form with respect to $X=(t, x)$

$$
\left.\begin{array}{r}
\operatorname{div}_{x} \mathbf{u}^{T}=0, \\
\partial_{t} \mathbf{u}+\operatorname{div}_{x}(\mathbf{u} \otimes \mathbf{u}+p \mathbb{I})=0 .
\end{array}\right\}
$$

By multiplying (26) with $\mathcal{B}(p, \mathbf{u})=\left(p-1 / 2|\mathbf{u}|^{2}, \mathbf{u}^{T}\right)$ we obtain the conservation law for the energy

$$
\partial_{t}\left(\frac{1}{2}|\mathbf{u}|^{2}\right)+\operatorname{div}_{x}\left(\frac{1}{2}|\mathbf{u}|^{2}+p \mathbf{u}^{T}\right)=0 .
$$

Corollaries 4.1, 4.2 and 4.3 imply that any weak solution $(p, \mathbf{u}) \in L^{3}(\mathscr{X}) \times$ $L^{3}\left(0, T ; B_{3, \infty}^{\alpha}\left(\mathbb{T}^{3}\right)\right)$ is a weak solution to $(27)$.

Remark. This result is comparable to [8]. 


\subsection{Compressible Euler System}

We consider the compressible Euler equations in the following form:

$$
\left.\begin{array}{r}
\partial_{t} \varrho+\operatorname{div}_{x}\left(\varrho \mathbf{u}^{T}\right)=0 \\
\partial_{t}(\varrho \mathbf{u})+\operatorname{div}_{x}(\varrho \mathbf{u} \otimes \mathbf{u}+p(\varrho) \mathbb{I})=0
\end{array}\right\} \quad \text { in } \mathscr{X}
$$

for an unknown vector field $\mathbf{u}: \mathscr{X} \rightarrow \mathbb{R}^{3}$ and scalar $\varrho: \mathscr{X} \rightarrow \mathbb{R}$. The function $p:[0, \infty) \rightarrow \mathbb{R}$ is given. Let $P$ be the so-called pressure potential given by $P(\varrho)=$ $\varrho \int_{1}^{\varrho} \frac{p(z)}{z^{2}} d z$. To get the conservation of the energy, we multiply (28) with

$$
\mathcal{B}(\varrho, \mathbf{u})=\left(P^{\prime}(\varrho)-\frac{1}{2}|\mathbf{u}|^{2}, \mathbf{u}^{T}\right)
$$

and obtain

$$
\partial_{t}\left(\frac{1}{2} \varrho|\mathbf{u}|^{2}+P(\varrho)\right)+\operatorname{div}_{x}\left[\left(\frac{1}{2} \varrho|\mathbf{u}|^{2}+P(\varrho)+p(\varrho)\right) \mathbf{u}^{T}\right]=0 .
$$

Let $(\varrho, \mathbf{u}) \in L^{3}\left(0, T ; B_{3, \infty}^{\alpha}\left(\mathbb{T}^{3}\right)\right) \times L^{3}\left(0, T ; B_{3, \infty}^{\alpha}\left(\mathbb{T}^{3} ; \mathbb{R}^{3}\right)\right)$ be a weak solution to (28) such that $\varrho \in[\varrho, \varrho]$ for some $0<\varrho<\bar{\varrho}<\infty$ and $\mathbf{u} \in B(0, R)$ for some $R>0$. Moreover, if $p \in C^{2}([\varrho, \bar{\varrho}])$, we use Corollary 4.1 to show that $(\varrho, \mathbf{u})$ is a weak solution to (29). ${ }^{1}$ In the contrast with the incompressible case, the continuity equation [the first equation of (28)] is not linear with respect to $\varrho$ and $\mathbf{u}$. Therefore, we have to assume that $\mathbf{u}$ is bounded to provide $\mathcal{B}(\varrho, \mathbf{u})$ is Lipschitz continuous in the range of $(\varrho, \mathbf{u})$.

Remark. If $\varrho>0$, system (28) can be rewritten with respect to the quantities $\varrho$ and $\mathbf{m}=\varrho \mathbf{u}$ as follows:

$$
\left.\begin{array}{r}
\partial_{t} \varrho+\operatorname{div}_{x}(\mathbf{m})=0 \\
\partial_{t} \mathbf{m}+\operatorname{div}_{x}\left(\frac{\mathbf{m} \otimes \mathbf{m}}{\varrho}+p(\varrho) \mathbb{I}\right)=0
\end{array}\right\} \quad \text { in } \mathscr{X} .
$$

A suitable choice of $\mathcal{B}$ is then

$$
\mathcal{B}(\varrho, \mathbf{m})=\left(P^{\prime}(\varrho)-\frac{|\mathbf{m}|^{2}}{2 \varrho^{2}}, \frac{\mathbf{m}^{T}}{\varrho}\right),
$$

which leads to the companion law

$$
\partial_{t}\left(\frac{|\mathbf{m}|^{2}}{2 \varrho}+P(\varrho)\right)+\operatorname{div}_{x}\left[\left(\frac{|\mathbf{m}|^{2}}{2 \varrho}+P(\varrho)+p(\varrho)\right) \mathbf{u}\right]=0 .
$$

As the continuity equation is now linear with respect to $(\varrho, \mathbf{m})$, we can apply Corollaries 4.1 and 4.3. As a consequence of this, a weak solution

$$
(\varrho, \mathbf{m}) \in L^{3}\left(0, T ; B_{3, \infty}^{\alpha}\left(\mathbb{T}^{3}\right)\right) \times L^{3}\left(0, T ; B_{3, \infty}^{\alpha}\left(\mathbb{T}^{3} ; \mathbb{R}^{3}\right)\right)
$$

such that $\varrho \in[\underline{\varrho}, \bar{\varrho}]$ for some $0<\varrho<\bar{\varrho}<\infty$ is also a weak solution to (32).

${ }^{1}$ We can extend $p$ from $[\underline{\varrho}, \bar{\varrho}]$ on $\mathbb{R}$ such that the extended function will be of class $C^{2}$ and compactly supported in $\mathbb{R}$. Moreover, due to the boundedness of $|\mathbf{u}|$ we can write $|\mathbf{u}|^{2}=\mathbf{u} \cdot T(\mathbf{u})$ in $\mathscr{X}$ where $T$ is a bounded Lipschitz function in $\mathbb{R}^{3}$. 


\subsection{Polyconvex Elasticity}

Let us consider the evolution equations of nonlinear elasticity, see e.g. [10] or [14],

$$
\left.\begin{array}{rl}
\partial_{t} F & =\nabla_{x} \mathbf{v} \\
\partial_{t} \mathbf{v} & =\operatorname{div}_{x}\left(D_{F} W(F)\right)
\end{array}\right\} \quad \text { in } \mathscr{X}
$$

for an unknown matrix field $F: \mathscr{X} \rightarrow \mathbb{M}^{k \times k}$, and an unknown vector field $\mathbf{v}: \mathscr{X} \rightarrow \mathbb{R}^{k}$. Function $W: \mathscr{U} \rightarrow \mathbb{R}$ is given. For many applications, $\mathscr{U}=\mathbb{M}_{+}^{k \times k}$ where $\mathbb{M}_{+}^{k \times k}$ denotes the subset of $\mathbb{M}^{k \times k}$ containing only matrices having positive determinant, see e.g. [1] for the discussion on the form of $W$ and $\mathscr{U}$. Let us point out that $\mathbb{M}_{+}^{k \times k}$ is a non-convex connected set.

System (33) can be rewritten in divergence form in $(t, x)$ as follows:

$$
\begin{aligned}
\partial_{t} F_{i, j} & =\partial_{x_{i}} u_{j}=\operatorname{div}_{x}\left(\left(\mathbf{e}^{i}\right)^{T} u_{j}\right), \quad \mathbf{e}_{j}^{i}=\delta_{i, j}, \\
\partial_{t} \mathbf{v} & =\operatorname{div}_{x}\left(D_{F} W(F)\right)^{T} .
\end{aligned}
$$

By considering $F$ with values in $\mathbb{R}^{k^{2}}$ and taking $\mathcal{B}(F, \mathbf{v})=\left(\left\{D_{F} W(F)\right\}^{T}, \mathbf{v}^{T}\right)$, we obtain the companion law

$$
\partial_{t}\left(\frac{1}{2}|\mathbf{v}|^{2}+W(F)\right)-\operatorname{div}\left(D_{F} W(F) \mathbf{v}\right)=0 .
$$

Let $(F, \mathbf{v}) \in B_{3, \infty}^{\alpha}\left(\mathscr{X} ; \mathbb{M}^{k \times k}\right) \times B_{3, \infty}^{\alpha}\left(\mathscr{X} ; \mathbb{R}^{3}\right)$ be a weak solution to (34) such that $F$ has a compact range in $\mathscr{U}$ and $\mathbf{v}$ in $\mathbb{R}^{k}$. Directly from Theorem $1.2,(F, \mathbf{v})$ is a weak solution to (35) whenever $W \in C^{3}(\mathscr{U})$.

Note that this observation for polyconvex elasticity is to the best of our knowledge, an original contribution.

\subsection{Magnetohydrodynamics}

Let us consider the system

$$
\left.\begin{array}{rl}
\operatorname{div}_{x} \mathbf{u}^{T} & =0 \\
\operatorname{div}_{x} \mathbf{h}^{T} & =0 \\
\partial_{t} \mathbf{u}+\left(\mathbf{u} \cdot \nabla_{x}\right) \mathbf{u}+\nabla_{x} p & =\left(\operatorname{curl}_{x} \mathbf{h}\right) \times \mathbf{h} \\
\partial_{t} \mathbf{h}+\operatorname{curl}_{x}(\mathbf{h} \times \mathbf{u}) & =0
\end{array}\right\} \text { in } \mathscr{X}
$$

for unknown vector functions $\mathbf{u}: \mathscr{X} \rightarrow \mathbb{R}^{3}$ and $\mathbf{h}: \mathscr{X} \rightarrow \mathbb{R}^{3}$ and an unknown scalar function $p: \mathscr{X} \rightarrow \mathbb{R}$. This system describes the motion of an ideal electrically conducting fluid, see e.g. [23, Chapter VIII]. Using standard vector calculus 
identities, (36) can be written in the divergence form as follows:

$$
\begin{aligned}
\operatorname{div}_{x} \mathbf{u}^{T} & =0, \\
\operatorname{div}_{x} \mathbf{h}^{T} & =0, \\
\partial_{t} \mathbf{u}+\operatorname{div}_{x}\left(\mathbf{u} \otimes \mathbf{u}+p \mathbb{I}+\frac{1}{2}|\mathbf{h}|^{2} \mathbb{I}-\mathbf{h} \otimes \mathbf{h}\right) & =0, \\
\partial_{t} \mathbf{h}+\operatorname{div}_{x}(\mathbf{h} \otimes \mathbf{u}-\mathbf{u} \otimes \mathbf{h}) & =0 .
\end{aligned}
$$

With $\mathcal{B}(p, \mathbf{u}, \mathbf{h})=\left(p-1 / 2|\mathbf{u}|^{2},-\mathbf{h} \cdot \mathbf{u}, \mathbf{u}^{T}, \mathbf{h}^{T}\right)$, the conservation of the total energy reads as

$$
\partial_{t}\left(\frac{1}{2}|\mathbf{u}|^{2}+\frac{1}{2}|\mathbf{h}|^{2}\right)+\operatorname{div}_{x}\left[\left(\frac{1}{2}|\mathbf{u}|^{2}+p+|\mathbf{h}|^{2}\right) \mathbf{u}^{T}-(\mathbf{u} \cdot \mathbf{h}) \mathbf{h}^{T}\right]=0
$$

A combination of Corollaries 4.1, 4.2 and 4.3 implies that any weak solution

$$
(p, \mathbf{u}, \mathbf{h}) \in L^{3}(\mathscr{X}) \times\left(L^{3}\left(0, T ; B_{3, \infty}^{\alpha}\left(\mathbb{T}^{3}\right)\right)\right)^{2}
$$

is a weak solution to (27). A similar result was obtained in [6].

\subsection{Further Examples}

The list of examples is still far from being complete, however it is not our goal to provide an extended list. Among the numerous possible further examples we will only mention inviscid compressible magnetohydrodynamics; a direct combination of Subsections 4.2 and 4.4 gives a sufficient condition for satisfying the relevant energy equality. Another example worth mentioning is heat conducting gas, see also [15].

Acknowledgements. This work was partially supported by the Simons-Foundation Grant 346300 and the Polish Government MNiSW 2015-2019 matching fund. PIOTR Gwiazda and Agnieszka Świerczewska-Gwiazda received support from the National Science Centre (Poland), 2015/18/MST1/00075. The research of MarTin MicháleK leading to these results has received funding from the European Research Council under the European Union's Seventh Framework Programme (FP7/2007-2013)/ ERC Grant Agreement 320078. The Institute of Mathematics of the Academy of Sciences of the Czech Republic is supported by RVO: 67985840 .

Open Access This article is distributed under the terms of the Creative Commons Attribution 4.0 International License (http://creativecommons.org/licenses/by/4.0/), which permits unrestricted use, distribution, and reproduction in any medium, provided you give appropriate credit to the original author(s) and the source, provide a link to the Creative Commons license, and indicate if changes were made. 


\section{References}

1. BALl, J.M.: Some open problems in elasticity. In: Geometry, Mechanics, and Dynamics, pages 3-59. Springer, New York, 2002.

2. Buckmaster, T.: Onsager's conjecture almost everywhere in time. Commun. Math. Phys., 333(3), 1175-1198, 2015

3. Buckmaster, T., De Lellis, C., Isett, P., SzéKelyhidi, L. JR.: Anomalous dissipation for 1/5-Hölder Euler flows. Ann. Math. (2), 182(1), 127-172, 2015

4. Buckmaster, T., De Lellis, C., SzéKelyhidi, L. JR.: Dissipative Euler flows with Onsager-critical spatial regularity. Commun. Pure Appl. Math., 69(9), 1613-1670, 2016

5. Buckmaster, T., De Lellis, C., SzéKelyhidi, L. JR., Vicol V.: Onsager's conjecture for admissible weak solutions. arXiv:1701.08678, 2017.

6. Caflisch, R.E., Klapper, I., Steele, G.: Remarks on singularities, dimension and energy dissipation for ideal hydrodynamics and MHD. Commun. Math. Phys., 184(2), 443-455, 1997

7. Cheskidov, A., Constantin, P., Friedlander, S., Shvydkoy, R.: Energy conservation and Onsager's conjecture for the Euler equations. Nonlinearity, 21(6), 1233-1252, 2008.

8. Constantin, P., E, W., Titi, E.S.: Onsager's conjecture on the energy conservation for solutions of Euler's equation. Commun. Math. Phys., 165(1), 207-209, 1994.

9. DAFERMos, C.M.: Hyperbolic conservation laws in continuum physics, volume 325 of Grundlehren der Mathematischen Wissenschaften [Fundamental Principles of Mathematical Sciences]. Springer, Berlin, fourth edition, 2016.

10. Dafermos, C.M., Hrusa, W.J.: Energy methods for quasilinear hyperbolic initialboundary value problems. Applications to elastodynamics. Arch. Ration. Mech. Anal., 87(3), 267-292, 1985.

11. De Lellis, C., SzéKelyhidi, L. JR.: The Euler equations as a differential inclusion. Ann. Math. (2), 170(3), 1417-1436, 2009.

12. De Lellis, C., SzÉKelyhidi, L. JR.: Dissipative continuous Euler flows. Invent. Math., 193(2), 377-407, 2013.

13. De Lellis, C., SzÉkelyhidi, L. JR.: Dissipative Euler flows and Onsager's conjecture. J. Eur. Math. Soc. (JEMS), 16(7), 1467-1505, 2014.

14. Demoulini, S., Stuart, D.M.A., Tzavaras, A.E.: A variational approximation scheme for three-dimensional elastodynamics with polyconvex energy. Arch. Ration. Mech. Anal., 157(4), 325-344, 2001.

15. Drivas, T.D., EyINK, G.L.: An Onsager singularity theorem for turbulent solutions of compressible Euler equations. Commu. Math. Phys. 2017. https://doi.org/10.1007/ s00220-017-3078-4.

16. Duchon, J., Robert, R.: Inertial energy dissipation for weak solutions of incompressible Euler and Navier-Stokes equations. Nonlinearity, 13(1), 249-255, 2000.

17. EyInK, G.L.: Energy dissipation without viscosity in ideal hydrodynamics. I. Fourier analysis and local energy transfer. Physica D, 78(3-4), 222-240, 1994.

18. Feireisl, E., Gwiazda, P., Świerczewska-Gwiazda, A., Wiedemann, E.: Regularity and energy conservation for the compressible Euler equations. Arch. Ration. Mech. Anal., 223(3), 1-21, 2017.

19. Godunov, S.K.: An interesting class of quasi-linear systems. Dokl. Akad. Nauk SSSR, 139, 521-523, 1961.

20. IseTt, P.: A Proof of Onsager's Conjecture.arXiv:1608.08301, 2016.

21. IsETT, P.: Hölder continuous Euler flows in three dimensions with compact support in time, volume 196 of Annals of Mathematics Studies. Princeton University Press, Princeton, NJ, 2017.

22. KANG, E., LEE, J.: Remarks on the magnetic helicity and energy conservation for ideal magnetohydrodynamics. Nonlinearity, 20(11), 2681-2689, 2007.

23. Landau, L.D., Lifshitz, E.M.: Electrodynamics of Continuous Media. Volume VIII of Course of Theoretical Physics. Pergamon Press, 1961. 
24. Leslie, T.M., Shvydroy, R.: The energy balance relation for weak solutions of the density-dependent Navier-Stokes equations. J. Differ. Equ., 261(6), 3719-3733, 2016.

25. Shvydkoy, R.: On the energy of inviscid singular flows. J. Math. Anal. Appl., 349(2), 583-595, 2009.

26. Shvydkoy, R.: Lectures on the Onsager conjecture. Discrete Contin. Dyn. Syst. Ser. S, 3(3), 473-496, 2010.

27. Yu C.: Energy conservation for the weak solutions of the compressible Navier-Stokes equations. Arch. Ration. Mech. Anal., 225(2), 1073-1087, 2017.

\section{Piotr Gwiazda}

Institute of Mathematics, Polish Academy of Sciences,

Śniadeckich 8,

Warsaw,

00-656 Poland.

e-mail:pgwiazda@mimuw.edu.pl

and

\section{Martin MicháLeK}

Institute of Mathematics of the Academy of Sciences of the Czech Republic, Žitná 25,

11567 Praha 1,

Czech Republic.

e-mail:michalek@math.cas.cz

and

AgnieszKa ŚwierczewsKa-Gwiazda

Institute of Applied Mathematics and Mechanics, University of Warsaw, Banacha 2, Warsaw, 02-097 Poland.

e-mail: aswiercz@mimuw.edu.pl

(Received June 30, 2017 / Accepted March 7, 2018)

Published online March 23, 2018

(C) The Author(s) (2018) 